\title{
Brendecke, Arndt, Friedrich, Markus, Friedrich, Susanne, Information in der Frühen Neuzeit. Status, Bestände, Strategien
}

Guillaume Garner

\section{(2) OpenEdition}

Journals

Édition électronique

URL : http://journals.openedition.org/ifha/6634

DOI : $10.4000 /$ ifha.6634

ISSN : 2198-8943

Éditeur

IFRA - Institut franco-allemand (sciences historiques et sociales)

Référence électronique

Guillaume Garner, «Brendecke, Arndt, Friedrich, Markus, Friedrich, Susanne, Information in der Frühen Neuzeit. Status, Bestände, Strategien », Revue de l'IFHA [En ligne], Date de recension, mis en ligne le 01 janvier 2011, consulté le 22 septembre 2020. URL : http://journals.openedition.org/ifha/6634 ; DOI : https://doi.org/10.4000/ifha.6634

Ce document a été généré automatiquement le 22 septembre 2020.

(C)IFHA 


\title{
Brendecke, Arndt, Friedrich, Markus, Friedrich, Susanne, Information in der Frühen Neuzeit. Status, Bestände, Strategien
}

\author{
Guillaume Garner
}

1 Ce volume collectif se situe à l'intersection de deux problématiques actuellement très prisées en Allemagne, celles de l'histoire du savoir (centrée sur les procédures et les pratiques concrètes plus que sur les contenus) et des procédures de communication comme clé de compréhension des structures sociales modernes. Au-delà du titre, très général, le propos de ces études est de mieux cerner le rapport entre savoir et pouvoir à travers la notion d'information. A.B., M.F. et S.F. définissent cette dernière comme une représentation du monde disponible en vue d'une mission précise, cette approche volontairement générale et ouverte permettant d'élargir le spectre des thèmes abordés, au risque de nuire parfois à l'homogénéité du volume. En particulier, la question du rapport entre " information » et " savoir » reste finalement peu abordée, malgré les propositions de P. Burke (qui voit dans la première le matériau de base du second, selon l'opposition entre le « cru » et le " cuit ») ou de L. Behrisch pour qui la transmission d'éléments de savoir est fondamentale dans le processus d'information. Mais ces différences sont aussi dues au fait que le terme d'information désigne à la fois un processus (la transmission de données) et ces données elles-mêmes, et que certaines contributions s'intéressent manifestement à l'une plus qu'à l'autre de ces dimensions.

2 Les rapports entre information et pouvoir sont donc abordés à travers une série d'études de cas concrètes portant sur les structures de pouvoir religieuses, qu'il s'agisse de la réforme de la Curie romaine en 1497, étudiée par J. Dendorfer, ou des débats au sein de l'ordre des jésuites sur le rôle des rapports écrits transmis aux autorités centrales de l'ordre (M. Friedrich). Le rapport entre information et construction de l'État moderne est évidemment abordé, ainsi par A. Brendecke à propos de la collecte d'informations dans l'Empire espagnol au XVIe siècle, ou par A. Holenstein qui souligne 
à quel point la collecte d'informations peut impliquer les populations concernées et s'intégrer ainsi dans une culture de la communication politique typique de la " police " d'Ancien Régime. Les administrations financières sont également étudiées par W. Schulze (à propos de la matricule d'Empire au XVIe siècle) et M. Hengerer (les processus d'information dans l'administration des finances des Habsbourg aux XVIe et XVIIe siècles). Le rôle du pouvoir politique comme initiateur d'informations cartographiques dans l'Empire est étudié par S. Friedrich, tandis que W.E.J. Weber souligne la signification croissante de l'information dans la science politique du XVIIe siècle. L'information "statistique » occupe, on l'aura compris, une place centrale dans ces questionnements : la dimension théorique de cette question est abordée par J. Nipperdey qui souligne les affinités entre les traités allemands de la fin du XVIIe siècle et le programme de l'arithmétique politique anglaise, tandis que les aspects pratiques sont étudiés par G. Gerber-Visser à propos de la Société économique de Berne au XVIIIe siècle comme acteur privé de collecte d'informations économiques, et par P. Becker qui propose une approche culturaliste (fortement marquée par les travaux de Bruno Latour) des pratiques de recensement démographique dans les États Habsbourg à la fin du XVIIIe siècle. Les acteurs impliqués dans le processus d'information, et notamment les réseaux de pouvoir ou de clientèle, sont également au centre de certains textes : A. Brendecke souligne le poids de ces réseaux dans l'empire espagnol tandis que J. Soll retrace les efforts de Colbert pour élaborer un monopole de l'information d'État, dirigé notamment contre les familles rivales du clan Colbert.

La perspective de la construction de l'État moderne (Staatsbildung) est plus marginale dans certaines interventions portant sur les bureaux d'adresses en Europe au XVIIIe siècle (A. Tantner) ou sur la collecte d'informations de nature " ethnographique » dans des traités de science du cérémoniel destinés à l'éducation de la jeunesse nobiliaire au tournant des XVIIe et XVIIIe siècles (M. Füssel), tandis que les deux dernières contributions opèrent une remise en perspective : $C$. Zwierlein examine la capacité des modèles évolutionnistes à interpréter l'évolution dans le temps long de la communication d'informations, tandis que L. Behrisch défend la thèse selon laquelle l'information est une procédure précise de traitement du savoir, à savoir sa transmission, sa communication, ce qui implique un ensemble de pratiques concrètes (standardisation, questionnaires, tableaux, etc.) que les trois directeurs de cet ouvrage se proposaient d'intégrer au questionnement des rapports entre savoir et pouvoir.

Par l'ampleur des thématiques abordées comme par les nombreuses études de cas concrètes proposées, ce volume est susceptible d'intéresser un large lectorat qui y trouvera des contributions solidement informées, stimulantes, et toutes agrémentées d'une substantielle bibliographie.

Guillaume Garner (ENS Lyon) 